\title{
An ACOUSTIC TRACTOR BEAM
}

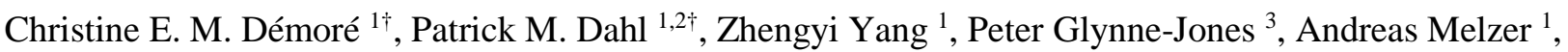 \\ Sandy Cochran ${ }^{1}$, Michael P. MacDonald ${ }^{1,4}$, Gabriel C. Spalding ${ }^{2 *}$ \\ ${ }^{1}$ Institute for Medical Science and Technology, University of Dundee, 1 Wurzburg Loan, Dundee, \\ Scotland, DD2 1FD, UK \\ ${ }^{2}$ Department of Physics, Illinois Wesleyan University, 201 E. Beecher Street, Bloomington, IL, \\ 61701, USA \\ ${ }^{3}$ Engineering Sciences, University of Southampton, University Road, Southampton, SO17 1BJ, UK \\ ${ }^{4}$ Division of Physics, University of Dundee, Nethergate, Dundee, Scotland, DD1 4HN, UK \\ ${ }^{\dagger}$ These authors contributed equally to the work \\ *Correspondence to: gspaldin@iwu.edu , mikepmacdonald@gmail.com
}

\section{PACS:}

43.25.Qp, Radiation pressure - acoustical

42.50.Wk, Light - Mechanical effects on atoms and molecules

43.20.El Sound - reflection, refraction, and diffraction of

43.20.Fn, Scattering - acoustical

Negative radiation forces act opposite to the direction of propagation, or net momentum, of a beam but have previously been challenging to definitively demonstrate. We report an experimental acoustic tractor beam generated by an ultrasonic array operating on macroscopic targets $(>1 \mathrm{~cm})$ to demonstrate the negative radiation forces, and to map out regimes over which they dominate, which we compare to simulations. The result and the geometrically simple configuration show that the effect is due to non-conservative forces, produced by redirection of a momentum flux from the angled sides of a target, and not by conservative forces from a potential energy gradient. Use of a simple acoustic setup provides an easily understood illustration of the negative radiation pressure concept for tractor beams, and demonstrates continuous attraction towards the source, against a net momentum flux in the system.

The momentum carried by fields and propagating waves has played a central role in the development of physics, impacting early discussions on the nature of light, the Second Law of Thermodynamics, the Stefan-Boltzmann law, and the development of Quantum Mechanics [1]. The association of this momentum with "action at a distance" has intrigued humankind for centuries, yielding science fiction concepts such as the "tractor beam," in which an outflow of energy results, somewhat counterintuitively, in an influx of matter.
Considering a general form of a tractor beam, theorists have recently proposed that attractive, or negative, forces can result from interactions of objects with directed optical and acoustic beams [2][11].

Some of the earliest experimental examples of remote manipulation with optical and acoustic fields took the form of levitation traps [12], [13], using positive, non-conservative, radiation pressure from a beam to push objects away from the source and balance against gravity. A positive radiation force $\left(F_{+}\right)$is relatively intuitive and is in reaction to either backscattering or absorption of the forward-directed momentum of a beam, and was famously reported in 1903 [14]. In contrast, most current optical and acoustic tweezing systems [15]-[18] are examples of conservative gradient force traps in which particles are drawn towards potential energy minima. However, tweezing systems that make use of potential energy wells do not provide the conceptual tractor beam defined in the theoretical literature, which is concerned with the role of (a negative) nonconservative radiation pressure, distinct from that of a gradient force, and directed towards the source. Time-evolving potential energy wells such as rotating anisotropic traps [19], or optical conveyors that move trapped particles by continuously sweeping potential energy minima [20], [21] are also not examples of non-conservative forces and hence do not constitute a tractor beam under this definition. Examples of (positive) non-conservative forces in 
optics and acoustics include the transfer of orbital angular momentum [22]-[25] or guided transport along Bessel beams [26]. Specifically, it has been proposed that a tractor beam involves an attractive (negative) non-conservative force upon a target; that is, a continuous redirection of momentum flux is required [4]-[8].

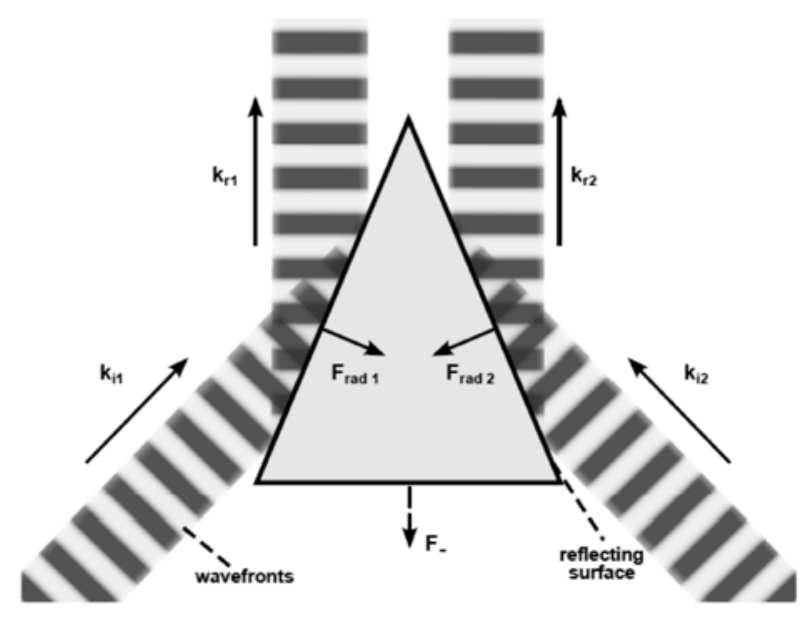

Fig. 1. Forward scattering of an acoustic or optical beam producing a net attraction force on a target. The change in momentum due to the axial redirection of a beam with locally off-axis components $\left(\mathrm{k}_{\mathrm{i} 1}, \mathrm{k}_{\mathrm{i} 2}\right)$ by reflection or scattering $\left(\mathrm{k}_{\mathrm{r} 1}, \mathrm{k}_{\mathrm{r} 2}\right)$ from the forward facing surfaces of an object results in a radiation force, $F_{\text {rad }}$, with negative axial components and a resultant negative radiation force, $F_{-}$, towards the source and opposite to the net momentum flux of the beam.

With complex beams, including those with conical or helical phase fronts [2], [8], [27]-[30], there can be a substantial reduction of the axial component of the local Poynting vector. It is the forward redirection of this locally off-axis "skew" momentum by a scattering object that leads to a negative radiation force, $F_{-}$, on the object, even as the net momentum flux, or net Poynting vector, of the beam remains directed away from the source. Fig. 1 illustrates such an arrangement and the concept of a tractor beam in its simplest form. Optical trapping systems demonstrating these principles have recently been reported, manipulating particles in the presence of a surface. In one approach, a tailored optical beam incident on microscopic particles in the presence of a reflecting surface produces both gradient and radiation forces; the targeted particles reach an equilibrium position where gradient forces balance a radiation pressure that is towards the virtual source [31]. In another approach, optical radiation forces opposing the projected axial momentum of an incident optical beam are exerted on particles situated at the interface between two different dielectric media when the beam is refracted towards the plane of the interface [32]. The experiment presented here demonstrates an acoustic negative radiation pressure directed towards the source, without the need for an additional reflecting surface or refractive interface. Moreover, since the region of $F_{\text {- }}$ extends from the source, providing a continuous attraction against a net momentum flux in the system, it is compatible with bringing samples in, from a distance, to docking contact with a source. The acoustic tractor beam is demonstrated with macroscopic samples (here $>1 \mathrm{~cm}$ ) since acoustic devices can generate significantly larger forces $(\mathrm{mN})$ than optical tweezers (pN) over larger length scales [25].

The present setup [33], illustrated in Fig. 2(a), uses a planar, 76-mm square aperture, ultrasonic matrix array operating at $550 \mathrm{kHz}$ to form a directed acoustic field in a water-filled chamber. The ultrasound system and matrix transducer array used for the experiments (ExAblate 2100, InSightec, Tirat Carmel, Israel) is a clinically-approved ultrasonic array system for MRI-guided focused ultrasound surgery [34], [35] with individually controllable transducer elements. The authors have previously demonstrated, with this system, that complex pressure fields such as high order helical beams [25] can be sculpted with appropriate control of the source aperture and phase profile. See Supplementary Material for more information on the array control. Using the matrix array system, we steer locally planar wavefronts towards the axis of symmetry to produce an acoustic field with a sinclike cross-sectional profile in the absence of a target (Fig. 2(b-d)). That is, we have produced rectilinear analogs of the cylindrically symmetric conical wavefronts associated with the Bessel-like beams discussed in much of the literature on negative radiation forces [2]-[8], [28]-[30]. This symmetric, rectilinear geometry simplifies implementation and characterization of the acoustic fields and the associated target design, and further demonstrates the ease with which negative radiation forces can be applied.

The phase profiles applied to the source array are designed to produce locally planar wavefronts steered at $50.6^{\circ}$ from rectangular source apertures, symmetric about the array centerline. By activating only a peripheral subset of the source array elements, we generate hollow-core beams with initial internal core size $\Delta x$. Simulated and measured maps of the pressure field transmitted from one side of the array (Fig. 2(b,d)) correspond well, showing 
the wavefronts steered at $\theta=50.6^{\circ}$ towards the centerline, and the grating lobe at $27^{\circ}$ away from the centerline, which does not interfere with the radiation force measurement. The simulated and measured interference patterns for the tractor beam in the absence of a target (Fig. 2(c,e)) show the expected distribution. As the pair of active apertures are stepped towards the centerline, reducing $\Delta x$, the $50.6^{\circ}$ steering angle and relative phases remain the same, and the region over which the wavefronts intersect moves closer to the source, as visualized in Movie S1 with Schlieren imaging [36].

In the presence of an appropriate target, scattering leads to redirection of the incoming wave, resulting in a continuous attractive force $(-\widehat{\mathbf{z}})$ towards the source when the beam intersects forward-facing sides of the target. Each target demonstrated here is a hollow isosceles triangular prism, extending the full length of the array, with an acoustic absorber on the base and thin metal sides to give large acoustic reflection coefficients. The targets were designed to demonstrate $F_{-}$and for mapping the force profile along the $z$-axis shown in Fig. 2. Target $A$ has an apex angle of $50^{\circ}$, approximately matching the steering angle to maximize the $+\hat{\boldsymbol{z}}$ change in momentum of the beam, while Target $\mathrm{B}$ has an apex angle of $38.2^{\circ}$, demonstrating that precise target geometry is not critical to the realization of $F_{-}$, and is smaller, allowing more localized force measurement and profile mapping (see Supplementary Material).

The net force on each target was measured directly as the excess or reduced weight on a balance from which the targets were suspended (see Supplementary Material). A force balance is the standard method for determining the power generated by clinical ultrasound equipment, measuring the positive radiation pressure on a target in the beam. For each active aperture with separation $\Delta x_{n}$, the force profile was measured as a function of the target position above the source, $z_{T}$. Finite element analysis [37] was used both to predict $F_{\text {net }}$, and to separate it into the component radiation forces on the target sides, $F_{-}$, and the base, $F_{+}$. Since acoustic velocity is not zero at all faces of the target, it is necessary to take this movement into account when calculating the mean force on the boundary. The net force on each surface was calculated via integration of the acoustic radiation stress tensor over the mean position of the surface of the target (see Supplementary Material). Measured and predicted force profiles are compared in Fig. 3, and a simulation of the interaction between the acoustic pressure field and Target $\mathrm{B}$ at different $z_{T}$ is visualized in Movie S2.
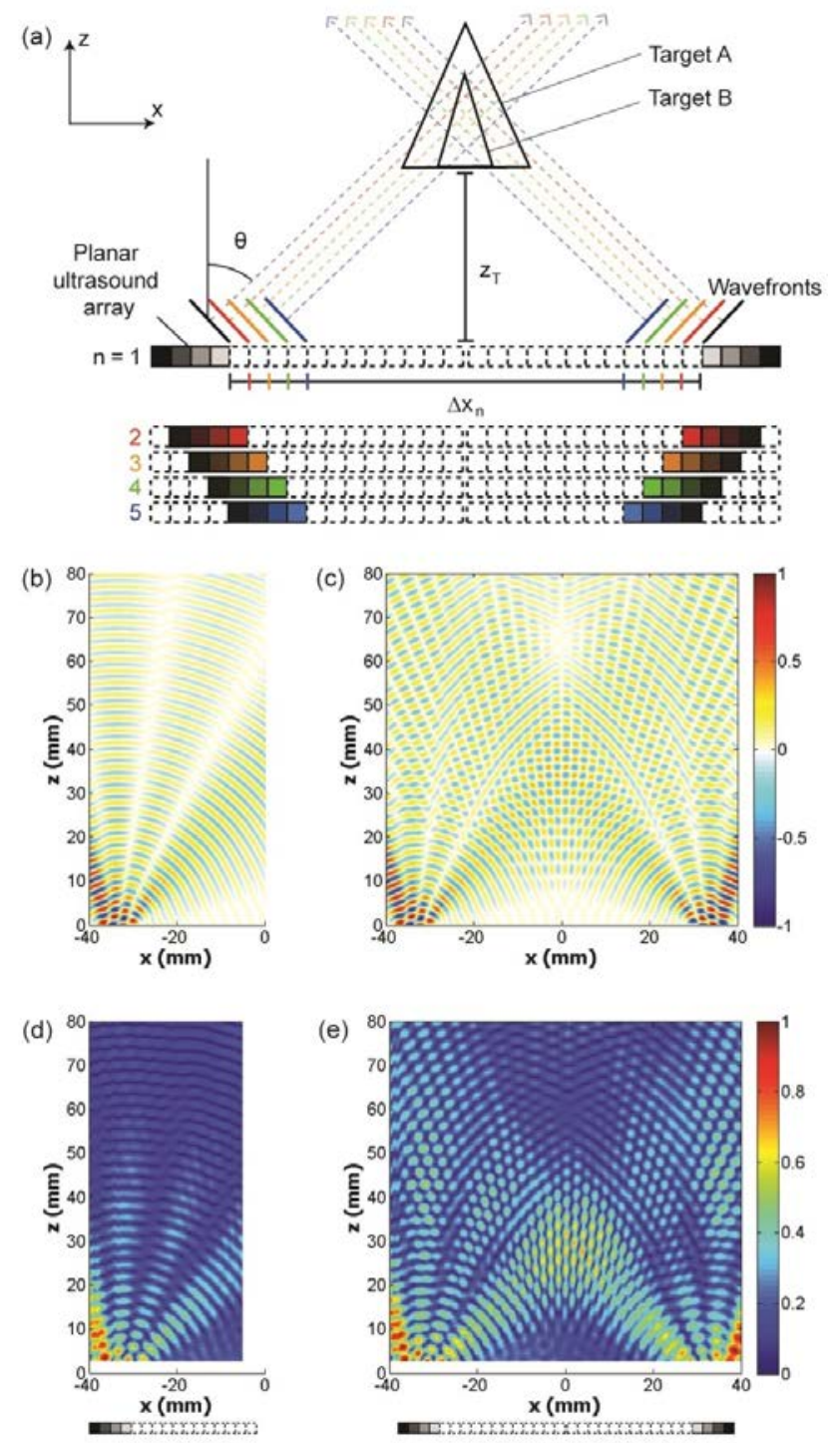

Fig. 2. Experimental configuration to demonstrate negative radiation forces with a planar ultrasonic array. (a) Scaled cross-sectional geometry of the $550 \mathrm{kHz}$ planar matrix array source, and hollow, prism-shaped targets suspended above the array. Linear phase gradients applied to the array elements produce wavefronts steered at $\theta=$ $50.6^{\circ}$ towards the array centerline. Active sub-apertures, forming a hollow core with diameter $\Delta x_{n}$, are stepped towards the centerline by the array element pitch, with a corresponding lateral $( \pm x)$ shift in the transmitted local wavefronts and an axial (-z), shift of the intersection with the axis. (b,c) Normalized maps of simulated instantaneous pressure field and (d,e) measured magnitude of the pressure field produced by the transmitting sub-apertures illustrated under the field maps.

The net steady state force depends simply on $Z_{T}$ and the cross-section of the wavefronts incident 
on the target. When the field is turned on, the net lateral forces on the sides of the target cause it to shift and rotate so its length is parallel with the centerline of the array, centering the target, no matter the initial lateral position. When the target is close to the array (small $z_{T}$ ), $F_{\text {net }}$ is indeed negative, pulling it towards the array because the steered wavefronts primarily interact with target side surfaces. As $z_{T}$ increases, the wavefronts begin to interact more with the absorbing base of the target and less with the sides, until $F_{+}$and $F_{-}$are balanced (Fig. 3A). When $z_{T}$ is big enough, the wavefronts are incident primarily on the base, so $F_{\text {net }}$ becomes positive, reaching a maximum upwards push when the base is at the highest intensity region of the field, before moving beyond the region of interference between the two crossing wavefronts. This same trend in forces is seen in both the simulation and experimental measurements, and for both targets.
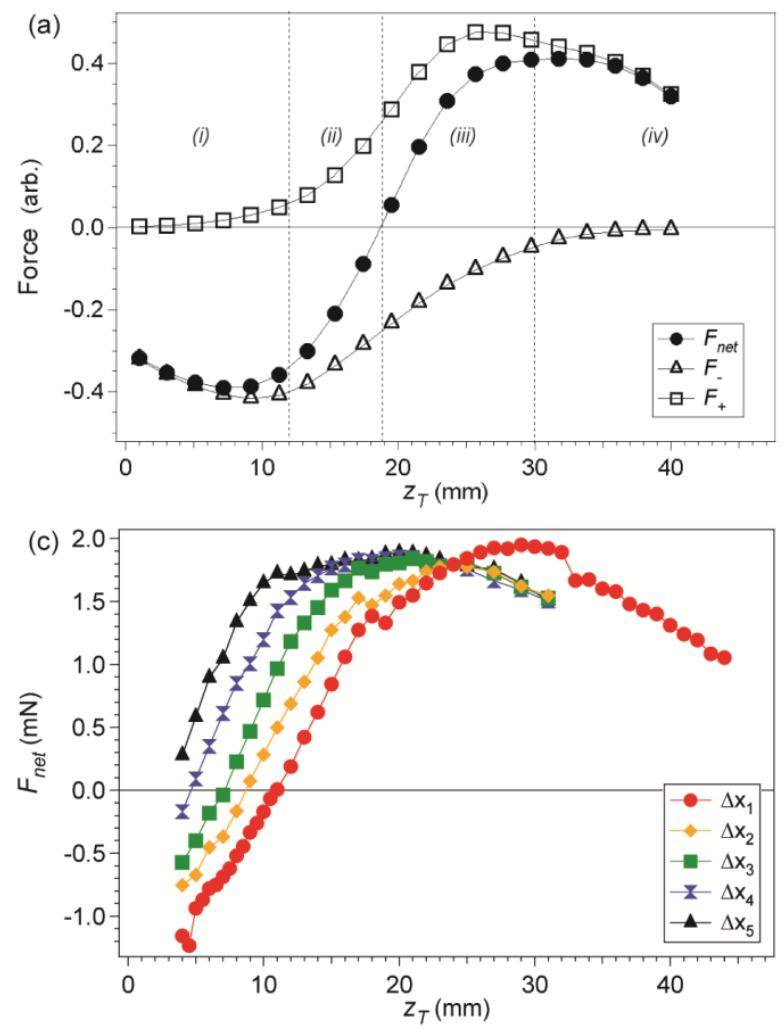

Fig. 3. (color online) Axial map of radiation forces on prism-shaped targets in tractor beams. (a) Predicted normalized net force profile, $F_{\text {net }}$, on Target B by field transmitted from active sub-apertures with separation $\Delta x_{1}$. $F_{\text {net }}$ is the sum of component vertical forces: positive radiation force, $F_{+}$, due to absorption on the base, and negative radiation force, $F_{-}$, from forward scattering at the target sides. Regions of interaction: (i) only the top of the target intersects the wavefronts and $F_{n e t}$ is minimized when wavefronts are maximally incident on target sides; (ii) wavefronts intersect more of the base and less of the sides until $F_{+}$balances $F_{-}$; (iii) wavefronts are primarily incident on the absorbing base, increasing then maximizing $F_{+}$; (iv) the target moves beyond the intersecting wavefronts and $F_{\text {net }}$ decreases. (b) Predicted normalized net forces on Target A and Target B, with varying separation between active array sub-apertures, $\Delta x_{n}$. Measured net forces on (c) Target A and (d) Target B showing the same trend as the predicted forces. Target $\mathrm{B}$ begins with larger $\mathrm{z}_{T}$ because experimental considerations required a thicker absorbing base.

The wavefronts intersect the broader base of Target A (Fig. 3(b,c)) at a lower $z$ than Target B (Fig. 3(b,d)), increasing the $F_{+}$component, and consequently $F_{\text {net }}$ is negative over a shorter distance from the source. However, the pulling force on Target A is bigger, up to $1 \mathrm{mN}$, because of the larger surface area and optimized apex angle compared to Target $B$. The difference in the ratio of the maximum upward and downward forces between simulation and experiment can be attributed to a decrease in $F_{-}$from the non-unitary reflection

( coefficient at the sides, and an increase in $F_{+}$from reflections at the imperfectly absorbing base of the target. As expected, reducing $\Delta x$ (Fig. 2(a)) has a similar effect to increasing $z_{T}$, such that the region, measured from the source, over which $F_{n e t}$ is negative, is shorter. With larger $\Delta x$, the maximum of $\left|F_{\text {net }}\right|$ reduces because of diverging and lower intensity fields farther from the source, and the position of maximum negative radiation force is farther from the source. For both predicted and measured forces, the axial position, $z_{T 0}$, at which $F_{\text {net }}=0$ (Fig. 4) increases linearly with $\Delta x$, corresponding to an axial shift in the interference field of the tractor beam. Differences between simulation and experiment can again be attributed to ₹ imperfect reflection and absorption by the targets. ह Above this position, the target is pushed away from $u$ the source, while below $z_{T 0}$, it is continuously pulled towards the source.

In this experiment, a phased array ultrasound source was used to apply a controllable negative radiation pressure that is continuous from the source until the directed beam diverges. The measured force profile confirms that the object is pulled towards the source even when the apex of the target intersects high intensity regions of the beam, demonstrating that the force is due to nonconservative radiation pressure, not a conservative force due to gradients in the field. These results also indicate that these methods, in addition to other techniques, extend the dexterity of an ultrasonic matrix array to the point of having the ability to 
acoustically manipulate the position of matter in all directions, given the proper phasing and drive. Using the present $76-\mathrm{mm}$ wide ultrasonic array, $F_{-}$has been demonstrated for objects centered up to $29 \mathrm{~mm}$ away. This suggests that a large aperture source is required to manipulate distant objects but it will be

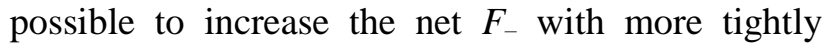
collimated beams, or more complex propagating beam types, such as Airy beams [38].

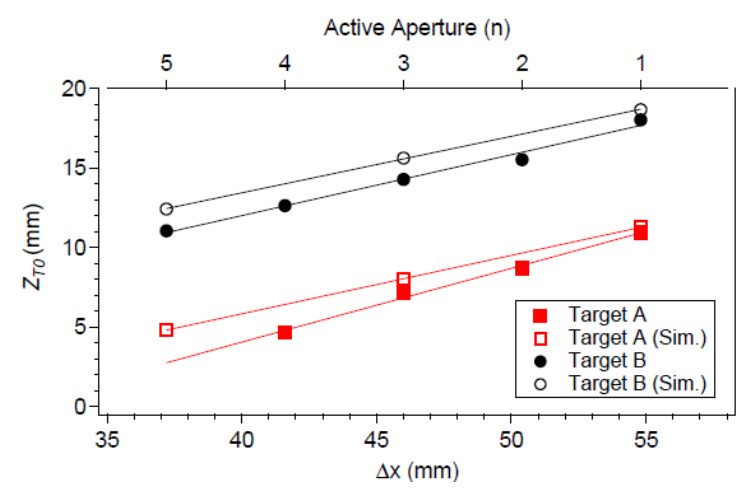

Fig. 4. (color online) Axial position of targets, $\mathrm{z}_{\mathrm{T} 0}$, at which positive and negative radiation forces balance, calculated from the zero crossings in Fig. 3 for both simulation and experimental measurement of $F_{\text {net. }}$.

Negative radiation forces on objects, which arise from the reflection or scattering of locally off-axis wavefronts towards the beam axis, have been proposed for a range of particle trapping and manipulation applications using both optical and acoustic beams. We have demonstrated experimentally negative acoustic radiation forces on macroscopic objects. The use of a clinically approved ultrasound system opens up a range of potential medical and biosciences applications that may exploit tailored and complex ultrasound beams. By implementing the advanced control of ultrasound fields developed in experiments such as this, there is significant potential to improve the control of energy deposition in focused ultrasound surgery and targeted drug delivery, in which high intensity beams are used to treat tumors noninvasively. Negative radiation forces might also be used for in vivo manipulation and stimulation of objects, fluids or biological tissue, yielding novel diagnostic techniques and treatment options. These, and other potential applications beyond the biomedical context in which the work was done, will benefit from the large forces possible with ultrasound, due to the long wavelength, and are not constrained to the simple target geometry used here. The depth penetration up to several centimeters we have achieved with the current ultrasound system is limited primarily by the discrete steering angles and the lateral extent of the current array. The approach demonstrated here provides additional incentives for developing tailored ultrasound fields for generating conservative and non-conservative forces, and adds to the set of techniques available for contact-free, dexterous manipulation of objects.

\section{Acknowledgments:}

This work has been funded by: the Engineering and Physical Science Research Council, UK, through the Electronic Sonotweezers research project (EPSRC EP/G01213X/1); the European Community's Seventh Framework Programme through the Nanoporation project (EU FP7 43915); and the European Regional Development Fund. The authors thank A. Volovick of InSightec Ltd., Y.Qiu and Z. Qiu of the University of Dundee, and the Sonotweezers project partners at the Universities of Bristol and Glasgow for their support and assistance in this research.

\section{Author Contributions}

GCS conceived the study, and with PMD, ZY, CEMD, SC and MPM, designed the experiments and analyzed the data. ZY and PMD performed the force measurements and pressure field measurements. PGJ, CEMD and PMD performed simulations. SC, MPM, and AM supervised the study and contributed experimental tools. PMD, CEMD, ZY, SC, MPM and GCS wrote the manuscript.

\section{Supplementary Movie Captions}

Movie S1. Schlieren imaging of the acoustic pressure fields generated with different active aperture separations, $\Delta x_{n}$, without a target in the water chamber.

http://youtu.be/2nnnguDnvQU

Movie S2. Simulation of the interaction between the acoustic pressure field and Target $B$ at different separations between source and target, $z_{T}$ using finite element analysis (PZFlex, Weidlinger Associates, Inc., CA, USA). The largest separation between the active apertures, $\Delta x_{1}$, is used in the simulation of the transmitted wavefronts.

\section{http://youtu.be/GlF4_zDzWK4}

\section{References and Notes:}

[1] B. Carazza and H. Kragh, "Adolfo Bartoli and the problem of radiant heat," Ann. Sci., vol. 46, no. 2, pp. 183-194, Mar. 1989.

[2] P. L. Marston, "Axial radiation force of a Bessel beam on a sphere and direction reversal of the force," J. Acoust. Soc. Am., vol. 120, no. 6, p. 3518, 2006.

[3] F. G. Mitri and Z. E. A. Fellah, "Theory of the acoustic radiation force exerted on a sphere by standing and quasistanding zero-order Bessel beam tweezers of variable half-cone angles.," IEEE Trans. Ultrason. Ferroelectr. Freq. Control, vol. 55, no. 11, pp. 2469-78, Nov. 2008.

[4] S. Sukhov and A. Dogariu, "On the concept of "tractor beams'," Opt. Lett., vol. 35, no. 22, p. 3847, Nov. 2010. 
[5] S. Sukhov and A. Dogariu, "Negative Nonconservative Forces: Optical 'Tractor Beams' for Arbitrary Objects," Phys. Rev. Lett., vol. 107, no. 20, p. 203602, Nov. 2011.

[6] J. Chen, J. Ng, Z. Lin, and C. T. Chan, "Optical pulling force," Nat. Photonics, vol. 5, no. 9, pp. 531-534, Jul. 2011.

[7] A. Novitsky, C.-W. Qiu, and H. Wang, "Single Gradientless Light Beam Drags Particles as Tractor Beams," Phys. Rev. Lett., vol. 107, no. 20, p. 203601, Nov. 2011.

[8] L. Zhang and P. L. Marston, "Geometrical interpretation of negative radiation forces of acoustical Bessel beams on spheres," Phys. Rev. E, vol. 84, no. 3, p. 35601, Sep. 2011.

[9] A. R. Zakharian, P. Polynkin, M. Mansuripur, and J. V Moloney, "Single-beam trapping of micro-beads in polarized light: Numerical simulations," Opt. Express, vol. 14, no. 8, p. 3660, Apr. 2006.

[10] J. Nemirovsky, M. C. Rechtsman, and M. Segev, "Negative radiation pressure and negative effective refractive index via dielectric birefringence," Opt. Express, vol. 20, no. 8, pp. 8907-8914, Apr. 2012.

[11] A. Dogariu, S. Sukhov, and J. Sáenz, "Optically induced 'negative forces'," Nat. Photonics, vol. 7, no. 1, pp. 24-27, Dec. 2012.

[12] A. Ashkin, "Acceleration and trapping of particles by radiation pressure," Phys. Rev. Lett., vol. 24, no. 4, pp. 156-159, Jan. 1970.

[13] A. Ashkin, "Optical Levitation by Radiation Pressure," Appl. Phys. Lett., vol. 19, no. 8, p. 283, 1971.

[14] E. F. Nichols and G. F. Hull, "The Pressure Due to Radiation. (Second Paper.)," Phys. Rev. (Series I), vol. 17, no. 1, pp. 26-50, Jul. 1903.

[15] A. Ashkin, J. M. Dziedzic, J. E. Bjorkholm, and S. Chu, "Observation of a single-beam gradient force optical trap for dielectric particles," Opt. Lett., vol. 11, no. 5, pp. 288290, 1986.

[16] D. G. Grier, "A revolution in optical manipulation.," Nature, vol. 424, no. 6950, pp. 810-6, Aug. 2003.

[17] K. C. Neuman and S. M. Block, "Optical trapping.," Rev. Sci. Instrum., vol. 75, no. 9, pp. 2787-809, Sep. 2004.

[18] M. Evander and J. Nilsson, "Acoustofluidics 20: applications in acoustic trapping.," Lab Chip, vol. 12, no. 22, pp. 4667-76, Nov. 2012.

[19] L. Paterson, M. P. MacDonald, J. Arlt, W. Sibbett, P. E. Bryant, and K. Dholakia, "Controlled rotation of optically trapped microscopic particles.," Science (80-. )., vol. 292, no. 5518, pp. 912-4, May 2001.

[20] T. Čižmár, V. Garcés-Chávez, K. Dholakia, and P. Zemánek, "Optical conveyor belt for delivery of submicron objects," Appl. Phys. Lett., vol. 86, no. 17, p. 174101, 2005.

[21] D. Ruffner and D. Grier, "Optical conveyors: A class of active tractor beams," Phys. Rev. Lett., vol. 109, no. 16, pp. 1-5, Oct. 2012.

[22] H. He, M. Friese, N. Heckenberg, and H. RubinszteinDunlop, "Direct Observation of Transfer of Angular Momentum to Absorptive Particles from a Laser Beam with a Phase Singularity," Phys. Rev. Lett., vol. 75, no. 5, pp. 826-829, Jul. 1995.

[23] J. Leach, S. Keen, M. J. Padgett, C. Saunter, and G. D. Love, "Direct measurement of the skew angle of the Poynting vector in a helically phased beam.," Opt. Express, vol. 14, no. 25, pp. 11919-24, Dec. 2006.

[24] K. Volke-Sepúlveda, A. Santillán, and R. Boullosa, "Transfer of Angular Momentum to Matter from Acoustical Vortices in Free Space," Phys. Rev. Lett., vol. 100, no. 2, pp. 2-5, Jan. 2008.

[25] C. E. M. Demore, Z. Yang, A. Volovick, S. Cochran, M. P. MacDonald, and G. C. Spalding, "Mechanical Evidence of the Orbital Angular Momentum to Energy Ratio of Vortex Beams," Phys. Rev. Lett., vol. 108, no. 19, p. 194301, May 2012.

[26] H. Little, C. T. A. Brown, V. Garcés-Chávez, W. Sibbett, and K. Dholakia, "Optical guiding of microscopic particles in femtosecond and continuous wave Bessel light beams," Opt. Express, vol. 12, no. 11, p. 2560, 2004.

[27] N. B. Simpson, D. McGloin, K. Dholakia, L. Allen, and M. J. Padgett, "Optical tweezers with increased axial trapping efficiency," J. Mod. Opt., vol. 45, no. 9, pp. 1943-1949, Sep. 1998.

[28] P. L. Marston, "Radiation force of a helicoidal Bessel beam on a sphere.," J. Acoust. Soc. Am., vol. 125, no. 6, pp. 3539-47, Jun. 2009.

[29] F. G. Mitri, "Negative axial radiation force on a fluid and elastic spheres illuminated by a high-order Bessel beam of progressive waves," J. Phys. A Math. Theor., vol. 42, no. 24, p. 245202, Jun. 2009.

[30] L. Zhang and P. L. Marston, "Axial radiation force exerted by general non-diffracting beams.," J. Acoust. Soc. Am., vol. 131, no. 4, pp. EL329-35, Apr. 2012.

[31] O. Brzobohatý, V. Karásek, M. Šiler, L. Chvátal, T. Čižmár, and P. Zemánek, "Experimental demonstration of optical transport, sorting and self-arrangement using a 'tractor beam'," Nat. Photonics, vol. 7, no. January, pp. 123-127, Jan. 2013.

[32] V. Kajorndejnukul, W. Ding, S. Sukhov, C.-W. Qiu, and A. Dogariu, "Linear momentum increase and negative optical forces at dielectric interface," Nat. Photonics, vol. 7, no. 10, pp. 787-790, Aug. 2013.

[33] See Supplemental Material at [URL will be inserted by publisher] for further details of the experimental setup, measurements and simulations.

[34] Y. Hertzberg, O. Naor, A. Volovick, and S. Shoham, "Towards multifocal ultrasonic neural stimulation: pattern generation algorithms.," J. Neural Eng., vol. 7, no. 5, p. 056002, Oct. 2010.

[35] Y. Hertzberg and G. Navon, "Bypassing absorbing objects in focused ultrasound using computer generated holographic technique," Med. Phys., vol. 38, no. 12, p. 6407, 2011.

[36] G. S. Settles, Schlieren and Shadowgraph Techniques. Berlin, Heidelberg: Springer Berlin Heidelberg, 2001.

[37] P. Glynne-Jones, P. P. Mishra, R. J. Boltryk, and M. Hill, "Efficient finite element modeling of radiation forces on elastic particles of arbitrary size and geometry.," $J$. Acoust. Soc. Am., vol. 133, no. 4, pp. 1885-93, Apr. 2013.

[38] K. Dholakia and T. Čižmár, "Shaping the future of manipulation," Nat. Photonics, vol. 5, no. 6, pp. 335-342, Jun. 2011. 